TRANSACTIONS OF THE

AMERICAN MATHEMATICAL SOCIETY

Volume 361, Number 7, July 2009, Pages 3915-3930

S 0002-9947(09)04678-9

Article electronically published on January 28, 2009

\title{
BROWNIAN SUBORDINATORS AND FRACTIONAL CAUCHY PROBLEMS
}

\author{
BORIS BAEUMER, MARK M. MEERSCHAERT, AND ERKAN NANE
}

\begin{abstract}
A Brownian time process is a Markov process subordinated to the absolute value of an independent one-dimensional Brownian motion. Its transition densities solve an initial value problem involving the square of the generator of the original Markov process. An apparently unrelated class of processes, emerging as the scaling limits of continuous time random walks, involves subordination to the inverse or hitting time process of a classical stable subordinator. The resulting densities solve fractional Cauchy problems, an extension that involves fractional derivatives in time. In this paper, we will show a close and unexpected connection between these two classes of processes and, consequently, an equivalence between these two families of partial differential equations.
\end{abstract}

\section{INTRODUCTION}

The goal of this paper is to establish a connection between two seemingly disparate classes of subordinated stochastic processes and their governing equations. The first class of processes uses Brownian motion as a subordinator 11, 2, 18, 19, 20, 21]. The second class uses the inverse or hitting time process of a nondecreasing stable Lévy process as a subordinator [38, 39, 40, 12, 41. One application of the Brownian subordinator involves scaling limits of transverse diffusion in a crack [21]. Inverse Lévy subordinators occur in scaling limits of continuous time random walks used to model anomalous diffusion in physics [43, 44, 61, finance [27, 42, 57, and hydrology [13, 15, 59. Neither class of processes is Markovian. However, their transition densities satisfy certain abstract differential equations that characterize the evolution of the process. Given a Markov process whose transition densities solve a Cauchy problem, a Brownian subordinator yields another process whose onedimensional distributions solve a related initial value problem involving the square of the generator of the Markov semigroup. Subordinating the same Markov process to an inverse stable Lévy process leads to a fractional Cauchy problem, where the integer time derivative is replaced by a fractional derivative whose order equals the stable index. Both situations lead to anomalous subdiffusion, where probability mass spreads slower than the classical $t^{1 / 2}$ rate seen in Brownian motion. In this paper we will show that, when the order of the fractional derivative equals $1 / 2$, the two governing equations have the same unique solution, and hence the two processes

Received by the editors June 26, 2007 and, in revised form, November 13, 2007.

2000 Mathematics Subject Classification. Primary 60J65, 60J60, 26A33.

Key words and phrases. Fractional diffusion, Lévy process, Cauchy problem, iterated Brownian motion, Brownian subordinator, Caputo derivative.

The second author was partially supported by NSF grant DMS-0417869.

(C)2009 American Mathematical Society Reverts to public domain 28 years from publication 
have the same one-dimensional distributions. Similar observations apply for other values of the fractional time derivative, and there are connections to subordination by a symmetric stable process. In applications to physics, the principal appeal of both classes is that the subordinators have a pleasant scaling property that can be inherited by the subordinated processes.

A Lévy process $B(t)$ has stationary, independent increments and $t \mapsto B(t)$ is continuous in probability; see, for example, Bertoin [16] or Sato [56. Classical Brownian motion in $\mathbb{R}^{d}$ is the special case where $B(t)$ has a mean zero normal distribution. In this case, the probability distribution $p(t, x)$ of $x=B(t)$ solves the diffusion equation $\partial p / \partial t=D \Delta p$ for some $D>0$, where $\Delta=\sum_{i} \partial^{2} / \partial x_{i}^{2}$. The random walk model of particle motion, where particles undergo independent identically distributed jumps at regularly spaced intervals in time, converges to Brownian motion as both the spatial and time scales increase to infinity. This close connection between Brownian motion and the diffusion equation is due to Bachelier [5] and Einstein [24]. Sokolov and Klafter [63] discuss modern extensions to include heavy tailed particle jumps and random waiting times, leading to fractional diffusion equations, the simplest of which is $\partial^{\beta} p / \partial t^{\beta}=-D(-\Delta)^{\alpha / 2} p$. The fractional Laplacian reflects heavy tailed particle jumps, where the probability of a jump magnitude exceeding some large $r>0$ diminishes like $r^{-\alpha}$, and the fractional time derivative codes heavy tailed waiting times, where the probability of waiting longer than $t$ falls off like $t^{-\beta}$. Heavy tails in space lead to superdiffusion, where a plume of particles spreads faster than the classical $t^{1 / 2}$ rate associated with Brownian motion. Heavy tails in time lead to subdiffusion, since long waiting times retard particle motion. See Metzler and Klafter 43, 44, for a recent survey.

A completely different model of particle motion emerges from the theory of random walks in random media, pioneered by Sinai 62. These models lead to interesting localization phenomena [25, 53] not seen in the case of random waiting times [17. A closely related problem is diffusion on fractals [7, 8, 9,. One particular application, diffusion in a crack, was led Burdzy and Khoshnevisan [21] to consider Brownian subordinators. In particular, a two-sided Brownian motion subordinated to an independent Brownian subordinator yields the scaling limit of transverse diffusion in a crack. Essentially, the crack is modeled using the sample path of the outer process, and the inner process or subordinator codes the subdiffusive effect of restricted motion in the crack. Given this history, it would be difficult to imagine that these two classes of non-Markovian processes exhibit any close connections. However, we will show that they are very closely related.

\section{BACKGROUND}

Let $B(t)$ be a Brownian motion on $\mathbb{R}^{d}$ and $Y_{t}$ another independent one-dimensional Brownian motion. Allouba and Zheng [1, 2, introduce a process they call Brownian time Brownian motion (BTBM) defined as $Z_{t}=B\left(\left|Y_{t}\right|\right)$. A related process called iterated Brownian motion process (IBM) was first considered by Burdzy [18. Take $B_{1}(t), B_{2}(t)$ to be independent Brownian motions on $\mathbb{R}^{d}$, and $Y_{t}$ as before. Define a two-sided Brownian motion by $B(t)=B_{1}(t)$ for $t \geq 0$ and $B_{2}(-t)$ for $t<0$. Then the IBM process is defined by $Z_{t}=B\left(Y_{t}\right)$. Various extensions of the BTBM or IBM have been considered, including a general Markov outer process [1, 2] and a symmetric stable subordinator studied by Nane [47, 48]. Similarly, the excursion-based Brownian-time process is defined by breaking up 
the path of $|Y(t)|$ into excursion intervals (i.e., maximal intervals of time on which $|Y(t)|>0)$ and, on each such interval, choosing an independent copy of the Markov process from a finite or an infinite collection. A simple conditioning argument shows that all these Brownian time processes have the same transition density functions, and hence the same one-dimensional distributions [2]. A similar remark holds for symmetric stable subordinators. That these processes are non-Markovian can be seen by noting that the transition densities do not solve the Kolmogorov equations.

Allouba and Zheng [2] and DeBlassie 23] show that for iterated Brownian motion (IBM) $Z_{t}=B\left(Y_{t}\right)$ the function

$$
u(t, x)=E_{x}\left[f\left(Z_{t}\right)\right]:=E\left[f\left(Z_{t}\right) \mid Z_{0}=x\right]
$$

solves the initial value problem

$$
\frac{\partial}{\partial t} u(t, x)=\frac{\Delta f(x)}{\sqrt{\pi t}}+\Delta^{2} u(t, x) ; \quad u(0, x)=f(x)
$$

for $t>0$ and $x \in \mathbb{R}^{d}$. The non-Markovian property of IBM is reflected in the appearance of the time-variable initial term in the PDE.

For a Markov process $X$, the family of linear operators $T(t) f(x)=E_{x}[f(X(t))]=$ $E[f(X(t)) \mid X(0)=x]$ forms a bounded continuous semigroup on the Banach space $L^{1}\left(\mathbb{R}^{d}\right)$, and the generator $L_{x} f(x)=\lim _{h \downarrow 0} h^{-1}(T(h) f(x)-f(x))$ is defined on a dense subset of that space; see, for example, 4, 28]. Then $u(t, x)=T(t) f(x)$ solves the Cauchy problem

$$
\frac{\partial}{\partial t} u(t, x)=L_{x} f(x) ; \quad u(0, x)=f(x)
$$

for $t>0$ and $x \in \mathbb{R}^{d}$. Allouba and Zheng 2] show that if we replace the outer process $B(t)$ with a continuous Markov process $X(t)$, the equation (2.1) holds, except that we replace the Laplacian in the PDE (2.1) with the generator $L_{x}$ of the continuous semigroup associated with this Markov process. That is, $u(t, x)=$ $E_{x}\left[f\left(Z_{t}\right)\right]$ solves the initial value problem

$$
\frac{\partial}{\partial t} u(t, x)=\frac{L_{x} f(x)}{\sqrt{\pi t}}+L_{x}{ }^{2} u(t, x) ; \quad u(0, x)=f(x)
$$

for $t>0$ and $x \in \mathbb{R}^{d}$.

Remark 2.1. Iterated Brownian motion (IBM) has many properties analogous to those of Brownian motion. The process $Z_{t}$ has stationary (but not independent) increments, and is self-similar with index $1 / 4$, meaning that $Z_{c t}$ and $c^{1 / 4} Z_{t}$ have the same finite-dimensional distributions for every $c>0$. Burdzy (1993) [18 showed that IBM satisfies a Law of the iterated logarithm (LIL):

$$
\limsup _{t \rightarrow \infty} \frac{Z_{t}}{t^{1 / 4}(\log \log (1 / t))^{3 / 4}}=\frac{2^{5 / 4}}{3^{3 / 4}} \quad \text { a.s. }
$$

A Chung-type LIL by was established by Khoshnevisan and Lewis 35] and Hu et al. 30. Khoshnevisan and Lewis [36] extended results of Burdzy [19] to develop a stochastic calculus for IBM. Local times of IBM were studied by Burdzy and Khosnevisan 20, Csáki et al. 22, Shi and Yor 60, Xiao 64, and $\mathrm{Hu}$ 29]. Bañuelos and DeBlassie 11] studied the distribution of the exit place for IBM in cones. Nane studied the lifetime asymptotics of IBM on bounded and unbounded domains [45, 46, 50], and generalized isoperimetric-type inequalities to IBM [49]. 
Remark 2.2. Funaki [26] considered a different version of iterated Brownian motion and studied the PDE connection of that process. Nane [4] established the PDE connection of a related class of processes defined in a similar manner, replacing the inner time process with a symmetric stable process.

Zaslavsky [65] introduced the fractional kinetic equation

$$
\frac{\partial^{\beta}}{\partial t^{\beta}} u(t, x)=L_{x} u(t, x) ; \quad u(0, x)=f(x)
$$

for Hamiltonian chaos, where $0<\beta<1$ and $L_{x}$ is the generator of some continuous Markov process $X_{0}(t)$ started at $x=0$. Here $\partial^{\beta} g(t) / \partial t^{\beta}$ is the Caputo fractional derivative in time, which can be defined as the inverse Laplace transform of $s^{\beta} \tilde{g}(s)-$ $s^{\beta-1} g(0)$, with $\tilde{g}(s)=\int_{0}^{\infty} e^{-s t} g(t) d t$ the usual Laplace transform. Baeumer and Meerschaert [10] and Meerschaert and Scheffler [40] show that the fractional Cauchy problem (2.4) is related to a certain class of subordinated stochastic processes. Take $D_{t}$ to be the stable subordinator, a Lévy process with strictly increasing sample paths such that $E\left[e^{-s D_{t}}\right]=e^{-t s^{\beta}}$; see, for example, [16, 56. Define the inverse or hitting time or first passage time process

$$
E_{t}=\inf \{x>0: D(x)>t\} .
$$

The subordinated process $Z_{t}=X_{0}\left(E_{t}\right)$ occurs as the scaling limit of a continuous time random walk (also called a renewal reward process), in which iid random jumps $\left\{Y_{i}\right\}$ are separated by iid positive waiting times $\left\{J_{i}\right\}$ [40. If the waiting times satisfy $P\left(J_{i}>t\right)=t^{-\beta} L(t)$, where $0<\beta<1$ and $L(t)$ is slowly varying, then they belong to the strict domain of attraction of a stable law with index $\beta$, and their partial sum process $S_{t}=J_{1}+\cdots+J_{[t]}$ converges after rescaling to the process $D_{t}$, in the Skorokhod $J_{1}$ topology on $D\left(\mathbb{R}^{+}, \mathbb{R}\right)$. The number of jumps by time $t>0$ is given by the inverse process $N_{t}=\max \left\{n: S_{n} \leq t\right\}$, and [40] shows that a rescaled version of $N_{t}$ converges to the hitting time process $E_{t}$ in the same topology. Similarly, if the iid vector particle jumps $\left\{Y_{i}\right\}$ satisfy a multivariable regular variation condition (or if they have a finite covariance matrix), then the partial sum process $V(t)=Y_{1}+\cdots+Y_{[t]}$ converges after linear operator rescaling to the operator Lévy motion $X_{0}(t)$, in the Skorokhod $J_{1}$ topology on $D\left(\mathbb{R}^{+}, \mathbb{R}^{d}\right)$; see [40, Theorem 4.1]. An operator Lévy motion is a Lévy process such that $X_{0}(t)$ has a centered operator stable distribution; see [37, Example 11.2.18]. This means that $X_{0}(c t)$ and $c^{E} X_{0}(t)$ are identically distributed for all $c>0$, for some linear operator $E$; see, for example, [34, 37. The continuous time random walk $V\left(S_{t}\right)$ models the location of a particle at time $t>0$. A continuous mapping argument, under some mild technical conditions, yields convergence of the rescaled CTRW to the subordinated process $X_{0}\left(E_{t}\right)$ in the somewhat weaker $M_{1}$ topology; see [40. Given a Banach space and a bounded continuous semigroup $T(t)$ on that space with generator $L_{x}$, it is well known that $p(t, x)=T(t) f(x)$ is the unique solution to the abstract Cauchy problem

$$
\frac{\partial}{\partial t} p(t, x)=L_{x} p(t, x) ; \quad p(0, x)=f(x)
$$

for any $f$ in the domain of $L_{x}$; see, for example, [4, 52. Theorem 3.1 in [10] shows that, in this setting, the formula

$$
u(t, x)=\int_{0}^{\infty} p\left((t / s)^{\beta}, x\right) g_{\beta}(s) d s
$$


yields the unique strong solution to the fractional Cauchy problem (2.4). Here $g_{\beta}(t)$ is the smooth density of the stable subordinator such that the Laplace transform $\tilde{g}_{\beta}(s)=\int_{0}^{\infty} e^{-s t} g_{\beta}(t) d t=e^{-s^{\beta}}$. Choose $x \in \mathbb{R}^{d}$ and let $X(t)=x+X_{0}(t)$. It follows from Theorem 5.1 in [40] that, in the special case where $T(t) f(x)=E_{x}[f(X(t))]$ is the semigroup on $L_{1}\left(\mathbb{R}^{d}\right)$ associated with the operator Lévy motion $X(t)$, the same formula (2.7) also equals $u(t, x)=E_{x}\left[f\left(Z_{t}\right)\right]$, where $Z_{t}=X\left(E_{t}\right)$ is the CTRW scaling limit process. Hence the subordinated process $Z_{t}$ is the stochastic solution to the fractional Cauchy problem (2.4) in this case.

In the case where $X_{0}(t)$ is a Lévy process started at zero and $X(t)=x+X_{0}(t)$ for $x \in \mathbb{R}^{d}$, the generator $L_{x}$ of the semigroup $T(t) f(x)=E_{x}[f(X(t))]$ is a pseudodifferential operator [3, 31, 58, that can be explicitly computed by inverting the Lévy representation. The Lévy process $X_{0}(t)$ has characteristic function

$$
E\left[\exp \left(i k \cdot X_{0}(t)\right)\right]=\exp (t \psi(k))
$$

with

$$
\psi(k)=i k \cdot a-\frac{1}{2} k \cdot Q k+\int_{y \neq 0}\left(e^{i k \cdot y}-1-\frac{i k \cdot y}{1+\|y\|^{2}}\right) \phi(d y),
$$

where $a \in \mathbb{R}^{d}, Q$ is a nonnegative definite matrix, and $\phi$ is a $\sigma$-finite Borel measure on $\mathbb{R}^{d}$ such that

$$
\int_{y \neq 0} \min \left\{1,\|y\|^{2}\right\} \phi(d y)<\infty
$$

see, for example, [37, Theorem 3.1.11] and [3, Theorem 1.2.14]. Let

$$
\hat{f}(k)=\int_{\mathbb{R}^{d}} e^{-i k \cdot x} f(x) d x
$$

denote the Fourier transform. Theorem 3.1 in [10] shows that $L_{x} f(x)$ is the inverse Fourier transform of $\psi(k) \hat{f}(k)$ for all $f \in D\left(L_{x}\right)$, where

$$
D\left(L_{x}\right)=\left\{f \in L^{1}\left(\mathbb{R}^{d}\right): \psi(k) \hat{f}(k)=\hat{h}(k) \exists h \in L^{1}\left(\mathbb{R}^{d}\right)\right\},
$$

and

$$
\begin{aligned}
L_{x} f(x) & =a \cdot \nabla f(x)+\frac{1}{2} \nabla \cdot Q \nabla f(x) \\
& +\int_{y \neq 0}\left(f(x+y)-f(x)-\frac{\nabla f(x) \cdot y}{1+y^{2}}\right) \phi(d y)
\end{aligned}
$$

for all $f \in W^{2,1}\left(\mathbb{R}^{d}\right)$, the Sobolev space of $L^{1}$-functions whose first and second partial derivatives are all $L^{1}$-functions. This includes the special case where $X_{0}(t)$ is an operator Lévy motion. We can also write $L_{x}=\psi(-i \nabla)$, where $\nabla=\left(\partial / \partial x_{1}, \ldots, \partial / \partial x_{d}\right)^{\prime}$. For example, if $X_{0}(t)$ is spherically symmetric stable, then $\psi(k)=-D\|k\|^{\alpha}$ and $L_{x}=-D(-\Delta)^{\alpha / 2}$, a fractional derivative in space, using the correspondence $k_{j} \rightarrow-i \partial / \partial x_{j}$ for $1 \leq j \leq d$. If $X_{0}$ has independent stable marginals, then one possible form is $\psi(k)=D \sum_{j}\left(i k_{j}\right)^{\alpha_{j}}$ and $L_{x}=D \sum_{j} \partial^{\alpha_{j}} / \partial x_{j}^{\alpha}$ using Riemann-Liouville fractional derivatives in each variable. This form does not coincide with the fractional Laplacian unless all $\alpha_{j}=2$.

Remark 2.3. The literature on fractional calculus uses a different semigroup definition. For example, in 10 the semigroup associated with a Lévy process $X_{0}(t)$ started at $x=0$ is defined by

$$
T_{F C}(t) f(x)=E\left[f\left(x-X_{0}(t)\right)\right]=\int_{\mathbb{R}^{d}} f(x-y) P_{X_{0}(t)}(d y) .
$$


One physical interpretation of this formula, when $f(x)$ is the probability density of a random variable $W$, is that $W$ represents the location of a randomly selected particle at time $t=0$, and $T_{F C}(t) f(x)$ is the probability density of the random particle location $W+X_{0}(t)$ at time $t>0$. In this paper we use the semigroup definition from the Markov process literature, based on the process $X(t)=x+X_{0}(t)$, so that

$$
T(t) f(x)=E_{x}[f(X(t))]=\int_{\mathbb{R}^{d}} f(x+y) P_{X_{0}(t)}(d y) .
$$

Clearly this is just a matter of replacing $X_{0}$ by $-X_{0}$. The paper [10] also uses a different definition $\hat{f}_{F C}(k)=\int e^{i k \cdot x} f(x) d x$ for the Fourier transform. Each of these two changes implies a change of $k$ to $-k$ in the formula for the Fourier transform of the semigroup, and hence the Fourier symbol $\psi(k)$ is the same for both. However, the interpretation of the Fourier symbol as a pseudo-differential operator changes. In the notation of this paper the derivative $\nabla f(x)$ has Fourier transform $(i k) \hat{f}(k)$, but in the notation of [10] the Fourier transform is $(-i k) \hat{f}(k)$. Hence the generator $L_{x}=\psi(i \nabla)$ in that paper, which explains why equation (2.8) differs from the corresponding formula (8) in [10].

Any Markov process semigroup operator $T(t) f(x)=E_{x}[f(X(t))]$ is a pseudodifferential operator

$$
T(t) f(x)=(2 \pi)^{-d} \int_{\mathbb{R}^{d}} e^{i k \cdot x} \lambda_{t}(x, k) \hat{f}(k) d k
$$

on the space of rapidly decreasing functions [33, Theorem 1.4]. Under some mild conditions, if the domain $D\left(L_{x}\right)$ of the generator of the extension of this semigroup to the Banach space of bounded continuous functions contains all smooth functions with compact support, then one can write

$$
\begin{aligned}
L_{x} f(x) & =a(x) \cdot \nabla f(x)+\frac{1}{2} \nabla \cdot Q(x) \nabla f(x) \\
& +\int_{y \neq 0}\left(f(x+y)-f(x)-\frac{\nabla f(x) \cdot y}{1+y^{2}}\right) \phi(d y, x),
\end{aligned}
$$

where $a(x) \in \mathbb{R}^{d}, Q(x)$ is a nonnegative definite matrix, and $\phi(d y, x)$ is a $\sigma$-finite Borel measure on $\mathbb{R}^{d}$ for each $x \in \mathbb{R}^{d}$ such that

$$
\int_{y \neq 0} \min \left\{1,\|y\|^{2}\right\} \phi(d y, x)<\infty ;
$$

see [32]. In this case, the Fourier symbol

$$
\psi(k, x)=i k \cdot a(x)-\frac{1}{2} k \cdot Q(x) k+\int_{y \neq 0}\left(e^{i k \cdot y}-1-\frac{i k \cdot y}{1+\|y\|^{2}}\right) \phi(d y, x),
$$

and $L_{x} f(x)=\psi(-i \nabla, x) f(x)$ is the inverse Fourier transform of $\psi(k, x) \hat{f}(k)$.

Remark 2.4. A connection between time-fractional equations and Brownian time was also noticed by Orsingher and Behgin [51, using a very different approach. They show that the density functions of iterated Brownian motion solve the onedimensional time-fractional equation $\partial^{1 / 2} u / \partial t^{1 / 2}=\partial^{2} u / \partial x^{2}$ by considering this equation as the end-member of a class of fractional telegraph equations. 


\section{MAIN RESUlts}

The results of this section establish a connection between two seemingly distinct classes of subordinated stochastic processes. Markov processes are stochastic solutions to the abstract Cauchy problem (2.6). Brownian subordinators yield stochastic solutions to an initial value problem (2.3) involving the square of the Markov generator. Inverse stable subordinators yield solutions to a fractional Cauchy problem (2.4) with the same Markov generator. In the case where the stable index $\beta=1 / 2$, our first results show that these two equations have the same solutions, and hence, the subordinated processes have the same one-dimensional distributions.

Theorem 3.1. Let $L_{x}$ be the generator of a continuous Markov semigroup $T(t) f(x)$ $=E_{x}\left[f\left(X_{t}\right)\right]$, and take $f \in D\left(L_{x}\right)$, the domain of the generator. Then, both the initial value problem (2.3) and the fractional Cauchy problem (2.4) with $\beta=1 / 2$ have the same solution given by

$$
u(t, x)=\frac{2}{\sqrt{4 \pi t}} \int_{0}^{\infty} T(s) f(x) \exp \left(-\frac{s^{2}}{4 t}\right) d s .
$$

Proof. Note that the Markov semigroup $T(t) f(x)=E_{x}\left[f\left(X_{t}\right)\right]$ is a uniformly bounded and strongly continuous semigroup on the Banach space $L^{1}\left(\mathbb{R}^{d}\right)$. Then Theorem 3.1 in Baeumer and Meerschaert [10] shows that the function $u(t, x)$ defined by (2.7) is the unique solution of the fractional Cauchy problem (2.4) for any $f \in D\left(L_{x}\right)$. In the case $\beta=1 / 2$, we have [3, Example 1.3.19]

$$
g_{1 / 2}(x)=\frac{1}{\sqrt{4 \pi x^{3}}} \exp \left(-\frac{1}{4 x}\right)
$$

and then a change of variables shows that

$$
u(t, x)=\frac{t}{\beta} \int_{0}^{\infty} p(s, x) g_{\beta}\left(\frac{t}{s^{1 / \beta}}\right) s^{-1 / \beta-1} d s=\int_{0}^{\infty} p(s, x) q(t, s) d s,
$$

where $p(t, x)=T(t) f(x)$ and

$$
\begin{aligned}
q(t, s)=2 t g_{1 / 2}\left(t / s^{2}\right) s^{-3} & =\frac{2 t}{s^{3} \sqrt{4 \pi t^{3} / s^{6}}} \exp \left(-\frac{s^{2}}{4 t}\right) \\
& =\frac{2}{\sqrt{4 \pi t}} \exp \left(-\frac{s^{2}}{4 t}\right)
\end{aligned}
$$

is a probability density on $s>0$ for all $t>0$. Hence we have that (3.1) is the unique solution to the fractional Cauchy problem (2.4).

Allouba and Zheng [2] show that the initial value problem (2.3) has the solution $u(t, x)=E_{x}\left[f\left(Z_{t}\right)\right]$, where $Z_{t}=B\left(\left|Y_{t}\right|\right)$ is the IBM process. It is not hard to check that the function $q(t, s)$ in $(3.3)$ is the probability density of the Brownian subordinator $\left|Y_{t}\right|$. Then a simple conditioning argument shows that

$$
u(t, x)=E_{x}\left[f\left(Z_{t}\right)\right]=\int_{0}^{\infty} q(t, s) p(s, x) d s,
$$

where $p(s, x)=T(s) f(x)=E_{x}\left[f\left(X_{t}\right)\right]$ is the unique solution to the initial value problem (2.6). Hence both the fractional Cauchy problem (2.4) and the initial value problem (2.3) have the same solution. 
Corollary 3.2. For $f \in D\left(\Delta_{x}\right)$, both the initial value problem

$$
\frac{\partial}{\partial t} u(t, x)=\frac{\Delta_{x} f(x)}{\sqrt{\pi t}}+\Delta_{x}^{2} u(t, x) ; \quad u(0, x)=f(x)
$$

and the fractional Cauchy problem

$$
\frac{\partial^{1 / 2}}{\partial t^{1 / 2}} u(t, x)=\Delta_{x} u(t, x) ; \quad u(0, x)=f(x)
$$

have the same solution given by (2.7), where

$$
p(t, x)=T(t) f(x)=\int_{\mathbb{R}^{d}} f(x-y)(4 \pi t)^{-d / 2} \exp \left(-\frac{\|y\|^{2}}{4 t}\right) d y .
$$

Proof. Apply Theorem 3.1 and note that (3.7) is the density of the Brownian motion Markov process with generator $\Delta_{x}$.

Suppose that $X(t)$ is an operator Lévy motion, $E_{t}=\inf \left\{x>0: D_{x}>t\right\}$ is the inverse or hitting time process of the stable subordinator $D_{t}$ with $E\left[e^{-s D_{t}}\right]=e^{-t s^{\beta}}$, and $Z_{t}=X\left(E_{t}\right)$. Let $L_{x}$ be the generator of the semigroup $T(t) f(x)=E_{x}[f(X(t))]$, a pseudo-differential operator defined by (2.8). It follows from Theorem 5.1 in [40] that, for any initial condition $f \in D\left(L_{x}\right)$, the function $u(t, x)=E_{x}\left[f\left(Z_{t}\right)\right]$ solves the fractional Cauchy problem (2.4). Hence we call the non-Markovian process $Z_{t}$ the stochastic solution to this abstract partial differential equation. The following theorem extends this result to a broader class of driving processes.

Theorem 3.3. For any continuous Markov semigroup $X(t)$ with generator $L_{x}$, let $E_{t}=\inf \left\{x>0: D_{x}>t\right\}$ be the inverse or hitting time process of the stable subordinator $D_{t}$, independent of $X$, with $E\left[e^{-s D_{t}}\right]=e^{-t s^{\beta}}$ for some $0<\beta<1$, and take $Z_{t}=X\left(E_{t}\right)$. Then for any initial condition $f \in D\left(L_{x}\right)$, the function $u(t, x)=E_{x}\left[f\left(Z_{t}\right)\right]$ solves the fractional Cauchy problem (2.4).

Proof. The proof is very similar to Theorem 3.1 above. Theorem 3.1 in [10 shows that the function $u(t, x)$ defined by (2.7) is the (unique) solution of the fractional Cauchy problem (2.4) for any $f \in D\left(L_{x}\right)$. A change of variables shows that (3.2) holds, where $g_{\beta}$ is the density of the random variable $D_{1}$. Corollary 3.1 in [40] shows that the function $q(t, s)=t \beta^{-1} g_{\beta}\left(s^{-1 / \beta} t\right) s^{-1 / \beta-1}$ is the density of the hitting time $E_{t}$, and then the result follows by a simple conditioning argument.

Corollary 3.4. For any continuous Markov process $X(t)$, both the Brownian-time subordinated process $X\left(\left|Y_{t}\right|\right)$ and the process $X\left(E_{t}\right)$ subordinated to the inverse $1 / 2$ stable subordinator have the same one-dimensional distributions. Hence they are both stochastic solutions to the fractional Cauchy problem (2.4), or, equivalently, to the higher order initial value problem (2.3).

Proof. In the proof of Theorem 3.1 we noted that the function $q(t, s)$ in (3.3) is the probability density of the Brownian subordinator $s=\left|Y_{t}\right|$, where $Y_{t}$ is a standard Brownian motion. The proof of Theorem 3.3 shows that this function is also the probability density of the inverse $1 / 2$-stable subordinator $s=E_{t}$. The result follows from a simple conditioning argument. 
Remark 3.5. The proof of Theorem 3.1 relies on Theorem 0.1 in Allouba and Zheng 2], but the proof of Theorem 0.1 in that paper may not be completely rigorous. The essential argument, using integration by parts twice, is that

$$
\begin{aligned}
\frac{\partial}{\partial t} u(t, x) & =\int_{0}^{\infty} T(s) f(x) \frac{\partial}{\partial t} q(t, s) d s \\
& =\int_{0}^{\infty} T(s) f(x) \frac{\partial^{2}}{\partial s^{2}} q(t, s) d s \\
& =\left.q(t, s) \frac{\partial}{\partial s}[T(s) f(x)]\right|_{s=0}+\int_{0}^{\infty} \frac{\partial^{2}}{\partial s^{2}}[T(s) f(x)] q(t, s) d s \\
& =q(t, 0) L_{x}[T(0) f(x)]+\int_{0}^{\infty} L_{x}^{2}[T(s) f(x)] q(t, s) d s \\
& =\frac{1}{\sqrt{\pi t}} L_{x} f(x)+L_{x}^{2} \int_{0}^{\infty} T(s) f(x) q(t, s) d s,
\end{aligned}
$$

which shows that (3.4) solves the higher order initial value problem (2.3). In a later paper 1, Allouba points out that the last step, where the operator $L_{x}$ is passed outside the integral, is not obvious and adds this as a technical condition, which is then verified in the special case $L_{x}=\Delta_{x}$, the Laplacian operator.

The next result is a restatement of Theorem 3.1 for Lévy semigroups. The proof does not use Theorem 0.1 in Allouba and Zheng [2], rather it relies on a LaplaceFourier transform argument. We will use the following notation for the Laplace, Fourier, and Fourier-Laplace transforms (respectively):

$$
\begin{aligned}
& \tilde{u}(s, x)=\int_{0}^{\infty} e^{-s t} u(t, x) d t, \\
& \hat{u}(t, k)=\int_{\mathbb{R}^{d}} e^{i k \cdot x} u(t, x) d x, \\
& \bar{u}(s, k)=\int_{\mathbb{R}^{d}} e^{i k \cdot x} \int_{0}^{\infty} e^{-s t} u(t, x) d t d x .
\end{aligned}
$$

Theorem 3.6. Suppose that $X(t)=x+X_{0}(t)$, where $X_{0}(t)$ is a Lévy process starting at zero. If $L_{x}$ is the generator (2.8) of the semigroup $T(t) f(x)=E_{x}\left[\left(f\left(X_{t}\right)\right)\right]$ on $L^{1}\left(\mathbb{R}^{d}\right)$, then, for any $f \in D\left(L_{x}\right)$, both the initial value problem (2.3) and the fractional Cauchy problem (2.4) with $\beta=1 / 2$ have the same unique solution given by (3.1).

Proof. Take Fourier transforms on both sides of (2.3) to get

$$
\frac{\partial \hat{u}(t, k)}{\partial t}=\frac{1}{\sqrt{\pi t}} \psi(k) \hat{f}(k)+\psi(k)^{2} \hat{u}(t, k),
$$

using the fact that $\psi(k) \hat{f}(k)$ is the Fourier transform of $L_{x} f(x)$. Then take Laplace transforms on both sides to get

$$
s \bar{u}(s, k)-\hat{u}(t=0, k)=s^{-1 / 2} \psi(k) \hat{f}(k)+\psi(k)^{2} \bar{u}(s, k),
$$

using the well-known Laplace transform formula

$$
\int_{0}^{\infty} \frac{t^{-\beta}}{\Gamma(1-\beta)} e^{-s t} d t=s^{\beta-1}
$$


for $\beta<1$. Since $\hat{u}(t=0, k)=\hat{f}(k)$, collecting like terms yields

$$
\bar{u}(s, k)=\frac{\left(1+s^{-1 / 2} \psi(k)\right) \hat{f}(k)}{s-\psi(k)^{2}}
$$

for $s>0$ sufficiently large.

On the other hand, taking Fourier transforms on both sides of (2.4) with $\beta=1 / 2$ gives

$$
\frac{\partial^{1 / 2} \hat{u}(t, k)}{\partial t^{1 / 2}}=\psi(k) \hat{u}(t, k) .
$$

Take Laplace transforms on both sides, using the fact that $s^{\beta} \tilde{g}(s)-s^{\beta-1} g(0)$ is the Laplace transform of the Caputo fractional derivative $\partial^{\beta} g(t) / \partial t^{\beta}$, to get

$$
s^{1 / 2} \bar{u}(s, k)-s^{-1 / 2} \hat{f}(k)=\psi(k) \bar{u}(s, k)
$$

and collect terms to obtain

$$
\begin{aligned}
\bar{u}(s, k) & =\frac{s^{-1 / 2} \hat{f}(k)}{s^{1 / 2}-\psi(k)} \\
& =\frac{s^{-1 / 2} \hat{f}(k)}{s^{1 / 2}-\psi(k)} \cdot \frac{s^{1 / 2}+\psi(k)}{s^{1 / 2}+\psi(k)} \\
& =\frac{\left(1+s^{-1 / 2} \psi(k)\right) \hat{f}(k)}{s-\psi(k)^{2}}
\end{aligned}
$$

which agrees with (3.9). For any fixed $k \in \mathbb{R}^{d}$, the two formulae are well-defined and equal for all $s>0$ sufficiently large.

Theorem 3.1 in Baeumer and Meerschaert [10] implies that the function $u(t, x)$ defined by (2.7) solves the fractional Cauchy problem (2.4) in $L^{1}\left(\mathbb{R}^{d}\right)$ for any $f \in$ $D\left(L_{x}\right)$. Take Fourier transforms in (2.7) and apply the Fubini theorem to get

$$
\hat{u}(t, k)=\int_{0}^{\infty} \hat{f}(k) \exp \left((t / s)^{1 / 2} \psi(k)\right) g_{1 / 2}(s) d s .
$$

Note that $\exp \left((t / s)^{1 / 2} \psi(k)\right)$ is bounded since $\psi(k)$ is negative definite, and then a simple dominated convergence argument shows that $\hat{u}(t, k)$ is continuous in $t>0$ for any $k \in \mathbb{R}^{d}$. Hence the uniqueness theorem for Laplace transforms 4, Theorem 1.7.3] shows that, for each $k \in \mathbb{R}^{d}$, (3.12) is the unique continuous function whose Laplace transform is given by either (3.9) or (3.11). Since $x \mapsto u(t, x)$ is an element of $L^{1}\left(\mathbb{R}^{d}\right)$ for every $t>0$, and since two elements of $L^{1}\left(\mathbb{R}^{d}\right)$ with the same Fourier transform are equal $d x$-almost everywhere, (3.1) is the unique element of $L^{1}\left(\mathbb{R}^{d}\right)$ whose Fourier transform equals (3.12).

Now if $u(t, x)$ is any solution to (2.3), then it has a Fourier-Laplace transform $\bar{u}(s, k)$ given by (3.9) or equivalently by (3.11). Since $\hat{u}(t, k)$ solves (3.8), it is differentiable in $t>0$ and hence continuous. Then the above argument shows that this solution is equal to (2.7) $d x$-almost everywhere for every $t>0$. Hence (2.7) solves both the higher order Cauchy problem (2.3), and the fractional Cauchy problem (2.4) with $\beta=1 / 2$, for any $f \in D\left(L_{x}\right)$, and it is the unique solution as an element of $L^{1}\left(\mathbb{R}^{d}\right)$, i.e., it is unique a.e.- $d x$ for every $t>0$. The proof of Theorem 3.1 shows that (2.7) reduces to (3.1) in this case. 
Remark 3.7. Uniqueness to (2.4) was shown by Bajlekova in [6, Corollary 3.2]; Allouba and Zheng [2] did not discuss uniqueness of solutions.

Remark 3.8. It is reasonable to conjecture that Theorem 3.6 can be extended to a sub-class of Markov processes $X(t)$ whose generators are pseudo-differential operators with negative definite symbols $\psi(k, x)$. One technical difficulty is that $\psi(k, x)^{n}$ is usually not the Fourier symbol of a semigroup generator for integers $n>1$.

The Fourier-Laplace transform method can be extended to establish connections between other fractional Cauchy problems and their corresponding higher order initial value problems. The next result gives one such correspondence.

Theorem 3.9. Suppose that $X(t)=x+X_{0}(t)$, where $X_{0}(t)$ is a Lévy process starting at zero. If $L_{x}$ is the generator (2.8) of the semigroup $T(t) f(x)=E_{x}\left[\left(f\left(X_{t}\right)\right)\right]$, then for any $f \in D\left(L_{x}\right)$, both the initial value problem

$$
\frac{\partial u(t, x)}{\partial t}=\frac{t^{-2 / 3}}{\Gamma(1 / 3)} L_{x} f(x)+\frac{t^{-1 / 3}}{\Gamma(2 / 3)} L_{x}^{2} f(x)+L_{x}^{3} u(t, x) ; \quad u(0, x)=f(x)
$$

and the fractional Cauchy problem (2.4) with $\beta=1 / 3$ have the same unique solution given by (2.7), where $g_{1 / 3}(t)$ is the probability density of the $1 / 3$-stable subordinator, so that $\int_{0}^{\infty} e^{-s t} g_{1 / 3}(t) d t=e^{-s^{1 / 3}}$ for all $s>0$.

Proof. The proof is very similar to Theorem 3.6. Take Fourier transforms on both sides of (3.13) to get

$$
\frac{\partial \hat{u}(t, k)}{\partial t}=\frac{t^{-2 / 3}}{\Gamma(1 / 3)} \psi(k) \hat{f}(k)+\frac{t^{-1 / 3}}{\Gamma(2 / 3)} \psi(k)^{2} \hat{f}(k)+\psi(k)^{3} \hat{u}(t, k) .
$$

Then take Laplace transforms on both sides to get

$$
s \bar{u}(s, k)-\hat{f}(k)=s^{-1 / 3} \psi(k) \hat{f}(k)+s^{-2 / 3} \psi(k)^{2} \hat{f}(k)+\psi(k)^{3} \bar{u}(s, k)
$$

and collect terms to obtain

$$
\bar{u}(s, k)=\frac{\left(1+s^{-1 / 3} \psi(k)+s^{-2 / 3} \psi(k)^{2}\right) \hat{f}(k)}{s-\psi(k)^{3}}
$$

for $s>0$ sufficiently large.

On the other hand, taking Fourier transforms on both sides of (2.4) with $\beta=1 / 3$ gives

$$
\frac{\partial^{\beta}}{\partial t^{\beta}} \hat{u}(t, k)=\psi(k) \hat{u}(t, k),
$$

and then taking Laplace transforms yields

$$
s^{1 / 3} \bar{u}(s, k)-s^{-2 / 3} \hat{f}(k)=\psi(k) \bar{u}(s, k) .
$$

Collecting terms yields

$$
\begin{aligned}
\bar{u}(s, k) & =\frac{s^{-2 / 3} \hat{f}(k)}{s^{1 / 3}-\psi(k)} \\
& =\frac{s^{-2 / 3} \hat{f}(k)}{s^{1 / 3}-\psi(k)} \cdot \frac{s^{2 / 3}+s^{1 / 3} \psi(k)+\psi(k)^{2}}{s^{2 / 3}+s^{1 / 3} \psi(k)+\psi(k)^{2}} \\
& =\frac{\left(1+s^{-1 / 3} \psi(k)+s^{-2 / 3} \psi(k)^{2}\right) \hat{f}(k)}{s-\psi(k)^{3}}
\end{aligned}
$$


which agrees with (3.14). For any fixed $k \in \mathbb{R}^{d}$, the two formulas are well-defined and equal for all $s>0$ sufficiently large. The remainder of the argument is identical to Theorem 3.6 .

Corollary 3.10. Suppose that $X(t)=x+X_{0}(t)$, where $X_{0}(t)$ is a Lévy process starting at zero, and let $E_{t}=\inf \left\{x>0: D_{x}>t\right\}$ be the inverse or hitting time process of the 1/3-stable subordinator $D_{t}$, independent of $X$, with $E\left[e^{-s D_{t}}\right]=$ $e^{-t s^{1 / 3}}$. If $L_{x}$ is the generator (2.8) of the semigroup $T(t) f(x)=E_{x}\left[\left(f\left(X_{t}\right)\right)\right]$ and $Z_{t}=X\left(E_{t}\right)$, then for any initial condition $f \in D\left(L_{x}\right)$, the function $u(t, x)=$ $E_{x}\left[f\left(Z_{t}\right)\right]$ solves the higher order initial value problem (3.13).

Proof. The corollary follows immediately from Theorem 3.9 together with Theorem 3.1 in 10 .

Remark 3.11. An easy extension of the argument for Theorem 3.9 shows that, under the same conditions, for any $n=2,3,4, \ldots$ both the initial value problem

$$
\frac{\partial u(t, x)}{\partial t}=\sum_{j=1}^{n-1} \frac{t^{1-j / n}}{\Gamma(j / n)} L_{x}^{j} f(x)+L_{x}^{n} u(t, x) ; \quad u(0, x)=f(x)
$$

and the fractional Cauchy problem (2.4) with $\beta=1 / n$ have the same unique solution given by (2.7) with $\beta=1 / n$. Hence the process $Z_{t}=X\left(E_{t}\right)$ is also the stochastic solution to this higher order initial value problem.

This paper has established a connection between processes subordinated to a Brownian time subordinator and to an inverse Lévy stable subordinator. A similar but weaker correspondence can also be established for stable time subordinators. Let $T(t) f(x)=E_{x}[f(X(t))]$ be the semigroup of a continuous Markov process $X(t)$ and let $L_{x}$ be its generator. Take $S_{t}$ a standard symmetric stable Lévy process with index $0<\alpha<2$, so that $E\left[e^{i k S(t)}\right]=e^{-t|k|^{\alpha}}$. Denote by $p(t, x)$ the density of $S(t)$, and let $Z_{t}=X\left(\left|S_{t}\right|\right)$. Nane [47] shows that if $\alpha=l / m$, where $l$ and $m$ are relatively prime, then $u(t, x)=E_{x}\left[f\left(Z_{t}\right)\right]$ solves

$$
(-1)^{l+1} \frac{\partial^{2 m}}{\partial t^{2 m}} u(t, x)=-\left.2 \sum_{i=1}^{l} \frac{\partial^{2 l-2 i}}{\partial x^{2 l-2 i}} p(t, x)\right|_{x=0} L_{x}^{2 i-1} f(x)-L_{x}^{2 l} u(t, x) ; \quad u(0, x)=f(x)
$$

for any bounded measurable function $f$ in the domain of $L_{x}$, with $D^{\gamma} f$ bounded and Hölder continuous for all multiindex $\gamma$ such that $|\gamma|=2 l$.

Suppose that $X(t)$ is a self-similar process with Hurst index $H$, so that $X(c t) \sim$ $c^{H} X(t)$ (equality in distribution). Then it is not hard to check that $Z_{t}=X\left(\left|S_{t}\right|\right)$ is also self-similar with $Z_{c t} \sim c^{H / \alpha} Z_{t}$. If $1<\alpha \leq 2$ and we take $E_{t}$ to be the inverse or hitting time process for a stable subordinator with index $\beta=1 / \alpha$, then $E_{c t} \sim c^{\beta} E_{t}$, and it follows that the process $X\left(E_{t}\right)$ is self-similar with the same index as $X\left(\left|S_{t}\right|\right)$. Corollary 3.1 in [40] shows that $E_{t}$ has moments of all orders, while the mean of $\left|S_{t}\right|^{\rho}$ diverges for $\rho>\alpha$. Hence it seems that these two processes are not equivalent.

Finally, we note that the equivalence established in this paper does not extend to strict subdomains of $\mathbb{R}^{d}$. Consider the Banach space $X=L^{1}\left(\mathbb{R}^{+}\right)$and the shift semigroup $[T(t) f](x):=f(x+t)$ with generator $L_{x} f=\frac{d}{d x} f$ and domain $\mathcal{D}\left(L_{x}\right)=\left\{f \in L^{1}\left(\mathbb{R}^{+}\right): f^{\prime} \in L^{1}\left(\mathbb{R}^{+}\right)\right\}$. In this case, solutions to the higher order 
initial value problem

$$
\frac{\partial}{\partial t} u(t, x)=\frac{L_{x} f(x)}{\sqrt{\pi t}}+L_{x}^{2} u(t, x) ; \quad u(0, x)=f(x) ; x \geq 0
$$

are not unique. To see this, note that if $u_{1}, u_{2}$ were any two solutions, then $u=$ $u_{1}-u_{2}$ would solve

$$
\frac{\partial}{\partial t} u(t, x)=L_{x}^{2} u(t, x) ; \quad u(0, x)=0, x \geq 0
$$

and uniqueness would require that $u \equiv 0$ is the only solution to (3.18). However,

$$
u(t, x)=\frac{1}{\sqrt{4 \pi t}} \exp \left(-\frac{(x+1)^{2}}{4 t}\right)
$$

solves (3.18) as well, so that solutions to (3.17) are not unique. On the other hand, solutions to the corresponding fractional Cauchy problem (2.4) with $\beta=$ $1 / 2$ are unique [6]. Hence the two forms are not equivalent on this domain. It is an interesting open problem to find governing partial differential equations for Brownian time processes on bounded subdomains. For a typical Markov process with generator $L_{x}$ on a bounded domain, one can solve the boundary value problem $\dot{u}=L_{x} u ; u=f$ on the boundary, as the expectation of $X(\tau)$ where $\tau$ is the hitting time at the boundary. DeBlassie 23 shows that the analogous result does not hold for iterated Brownian motion, and it is likely that a resolution of these problems will require a novel approach.

\section{REFERENCES}

[1] H. Allouba, Brownian-time processes: The pde connection and the corresponding FeynmanKac formula, Trans. Amer. Math. Soc. 354 (2002), no. 11 4627-4637. MR1926892 (2003m:60177)

[2] H. Allouba and W. Zheng, Brownian-time processes: The pde connection and the halfderivative generator, Ann. Prob. 29 (2001), no. 2, 1780-1795. MR1880242 (2002j:60118)

[3] D. Applebaum (2004) Lévy Processes and Stochastic Calculus. Cambridge studies in advanced mathematics. MR 2072890 (2005h:60003)

[4] W. Arendt, C. Batty, M. Hieber, and F. Neubrander, Vector-valued Laplace transforms and Cauchy problems. Monographs in Mathematics, Birkhäuser-Verlag, Berlin (2001). MR.1886588 (2003g:47072)

[5] L.J.B. Bachelier (1900) Théorie de la Spéculation. Gauthier-Villars, Paris.

[6] E.G. Bajlekova, Fractional evolution equations in Banach spaces, Ph.D. thesis, Eindhoven University of Technology, 2001. MR1868564 (2002h:34115)

[7] M.T. Barlow (1990) Random walks and diffusion on fractals. In Proceedings of the Internatioal Congress of Mathematicians, Kyoto, Japan 2, 1025-1035. Springer-Verlag. MR.1159287

[8] M.T. Barlow and R.F. Bass (1993) Coupling and Harnack inequalities for Sierpiński carpets. Bull. Amer. Math. Soc. 29 208-212. MR.1215306 (94a:60011)

[9] M.T. Barlow and E.A. Perkins (1988) Brownian motion on the Sierpiński carpet. Probab. Theory Rel. Fields 79 543-623. MR966175 (89g:60241)

[10] B. Baeumer and M.M. Meerschaert, Stochastic solutions for fractional Cauchy problems, Fractional Calculus Appl. Anal. 4 (2001), 481-500. MR1874479 (2003d:26006)

[11] R. Bañuelos and R.D. DeBlassie (2006), The exit distribution for iterated Brownian motion in cones. Stochastic Processes and their Applications 116 no. 1, 36-69. MR2186839 (2007k:60258)

[12] P. Becker-Kern, M.M. Meerschaert and H.P. Scheffler (2004) Limit theorems for coupled continuous time random walks. The Annals of Probability 32, No. 1B, 730-756. MR2039941 (2004m:60092)

[13] D. Benson, S. Wheatcraft and M. Meerschaert (2000) Application of a fractional advectiondispersion equation. Water Resour. Res. 36, 1403-1412. 
[14] D. Benson, S. Wheatcraft and M. Meerschaert (2000) The fractional-order governing equation of Lévy motion, Water Resources Research 36, 1413-1424.

[15] D. Benson, R. Schumer, M. Meerschaert and S. Wheatcraft (2001) Fractional dispersion, Lévy motions, and the MADE tracer tests. Transport in Porous Media 42, 211-240. MR.1948593

[16] J. Bertoin (1996) Lévy processes. Cambridge University Press. MR.1406564 (98e:60117)

[17] J. Bisquert (2003) Fractional Diffusion in the Multiple-Trapping Regime and Revision of the Equivalence with the Continuous-Time Random Walk. Physical Review Letters 91, No. 1, $602-605$.

[18] K. Burdzy, Some path properties of iterated Brownian motion, In Seminar on Stochastic Processes (E. Çinlar, K.L. Chung and M.J. Sharpe, eds.), Birkhäuser, Boston (1993), 67-87. MR.1278077 (95c:60075)

[19] K. Burdzy, Variation of iterated Brownian motion, In Workshops and Conference on Measurevalued Processes, Stochastic Partial Differential Equations and Interacting Particle Systems (D.A. Dawson, ed.) Amer. Math. Soc. Providence, RI (1994), 35-53. MR1278281(95h:60123)

[20] K. Burdzy and D. Khoshnevisan, The level set of iterated Brownian motion, Séminarie de probabilités XXIX (Eds.: J Azéma, M. Emery, P.-A. Meyer and M. Yor), Lecture Notes in Mathematics, 1613, Springer, Berlin (1995), 231-236. MR.1459464 (98k:60138)

[21] K. Burdzy and D. Khoshnevisan, Brownian motion in a Brownian crack, Ann. Appl. Probabl. 8 (1998), no. 3, 708-748. MR1627764 (99g:60147)

[22] E. Csáki, M. Csörgö, A. Földes, and P. Révész, The local time of iterated Brownian motion, J. Theoret. Probab. 9 (1996), 717-743. MR1400596 (97f:60173)

[23] R. D. DeBlassie, Iterated Brownian motion in an open set, Ann. Appl. Prob. 14 (2004), no. 3, 1529-1558. MR2071433 (2005f:60172)

[24] A. Einstein (1956) Investigations on the theory of Brownian movement. Dover, New York. MR0077443(17:1035g)

[25] L.R.G. Fontes, M. Isope and C.M. Newman (2002) Random walks with strongly inhomogeneous rates and singular diffusions: Convergence, localization, and aging in one dimension. Ann. Probab. 30, no. 2, 579-604. MR1905852 (2003e:60229)

[26] T. Funaki, A probabilistic construction of the solution of some higher order parabolic differential equations, Proc. Japan Acad. Ser. A. Math. Sci. 55 (1979), no. 5, 176-179. MR533542 (80h:60075)

[27] R. Gorenflo, F. Mainardi, E. Scalas and M. Raberto (2001) Fractional calculus and continuous-time finance. III. The diffusion limit. Mathematical finance (Konstanz, 2000), 171-180, Trends Math., Birkhäuser, Basel. MR1882830

[28] E. Hille and R.S. Phillips (1957) Functional Analysis and Semi-Groups. Amer. Math. Soc. Coll. Publ. 31, American Mathematical Society, Providence, RI. MR0089373 (19:664d)

[29] Y. Hu, Hausdorff and packing measures of the level sets of iterated Brownian motion. J. Theoret. Probab. 12 (1999), no. 2, 313-346. MR1684747 (2000f:60054)

[30] Y. Hu., D. Pierre-Loti-Viaud, and Z. Shi, Laws of iterated logarithm for iterated Wiener proceses, J. Theoret. Probabl. 8 (1995), 303-319. MR1325853 (96b:60073)

[31] N. Jacob (1996) Pseudo-Differential Operators and Markov Processes. Akad. Verlag, Berlin. MR1409607 (97m:60109)

[32] N. Jacob (1998) Characteristic functions and symbols in the theory of Feller processes. Potential Anal. 8, no. 1, 61-68. MR 1608650 (99b:60121)

[33] N. Jacob and R. Schilling (2001) Lévy-type processes and pseudodifferential operators. Lévy processes, 139-168, Birkhäuser, Boston, MA. MR.1833696 (2002c:60077)

[34] Z. Jurek and J.D. Mason (1993) Operator-Limit Distributions in Probability Theory. Wiley, New York. MR:1243181 (95b:60018)

[35] D. Khoshnevisan and T.M. Lewis, Stochastic calculus for Brownian motion in a Brownian fracture, Ann. Applied Probabl. 9 (1999), no. 3, 629-667. MR1722276 (2001m:60128)

[36] D. Khoshnevisan and T.M. Lewis, Chung's law of the iterated logarithm for iterated Brownian motion, Ann. Inst. H. Poincaré Probab. Statist. 32 (1996), no. 3, 349-359. MR.1387394 (97k:60218)

[37] M.M. Meerschaert and H.P. Scheffler (2001) Limit Distributions for Sums of Independent Random Vectors: Heavy Tails in Theory and Practice. Wiley Interscience, New York. MR:1840531(2002i:60047) 
[38] M.M. Meerschaert, D.A. Benson, H.P. Scheffler and B. Baeumer (2002) Stochastic solution of space-time fractional diffusion equations. Phys. Rev. E 65, 1103-1106. MR 1917983 (2003d:60165)

[39] M.M. Meerschaert, D.A. Benson, H.P. Scheffler and P. Becker-Kern (2002) Governing equations and solutions of anomalous random walk limits. Phys. Rev. E 66, 102R-105R.

[40] M.M. Meerschaert and H.P. Scheffler (2004) Limit theorems for continuous time random walks with infinite mean waiting times. J. Applied Probab. 41, no. 3, 623-638. MR2074812 (2005f:60105)

[41] M.M. Meerschaert and H.P. Scheffler (2006) Stochastic model for ultraslow diffusion. Stoch. Proc. Appl., 116, no. 9, 1215-1235. MR2251542

[42] M.M. Meerschaert and E. Scalas (2006) Coupled continuous time random walks in finance. Physica A, 370, 114-118. MR2263769 (2007e:91097)

[43] R. Metzler and J. Klafter (2000) The random walk's guide to anomalous diffusion: A fractional dynamics approach. Phys. Rep. 339, 1-77. MR1809268 (2001k:82082)

[44] R. Metzler and J. Klafter (2004) The restaurant at the end of the random walk: recent developments in the description of anomalous transport by fractional dynamics. J. Physics A 37, R161-R208. MR2090004

[45] E. Nane, Iterated Brownian motion in parabola-shaped domains, Potential Analysis, 24 (2006), 105-123. MR2217416

[46] E. Nane, Iterated Brownian motion in bounded domains in $\mathbb{R}^{n}$, Stochastic Processes and Their Applications 116 (2006), 905-916. MR2254664 (2007j:60133)

[47] E. Nane, Higher order PDE's and iterated processes, Transactions of American Mathematical Society 360 (2008), 2681-2692. MR2373329 (2008j:60202)

[48] E. Nane, Laws of the iterated logarithm for $\alpha$-time Brownian motion, Electron. J. Probab. 11 (2006), no. 18, 434-459 (electronic). MR2223043 (2007c:60087)

[49] E. Nane, Isoperimetric-type inequalities for iterated Brownian motion in $\mathbb{R}^{n}$, Statistics \& Probability Letters 78 (2008), 90-95. MR2381278 (2008k:60194)

[50] E. Nane, Lifetime asymptotics of iterated Brownian motion in $\mathbb{R}^{n}$, Esaim Probab. Stat., March 2007, Vol. 11, pp. 147-160. MR2299652 (2008a:60207)

[51] E. Orsingher and L. Beghin (2004) Time-fractional telegraph equations and telegraph processes with Brownian time. Prob. Theory Rel. Fields 128, 141-160. MR2027298 (2005a:60056)

[52] A. Pazy (1983) Semigroups of Linear Operators and Applications to Partial Differential equations. Applied Mathematical Sciences 44, Springer-Verlag, New York. MR710486 (85g:47061)

[53] T. Prosen and M. Znidaric (2001) Anomalous diffusion and dynamical localization in polygonal billiards. Phys. Rev. Lett. 87, 114101-114104.

[54] W. Rudin (1973) Functional Analysis. 2nd Edition, McGraw-Hill, New York. MR.0365062 $(51: 1315)$

[55] G. Samorodnitsky and M. Taqqu, Stable non-Gaussian Random processes, Chapman and Hall, New York (1994). MR1280932(95f:60024)

[56] K.I. Sato (1999) Lévy Processes and Infinitely Divisible Distributions. Cambridge University Press. MR1739520 (2003b:60064)

[57] E. Scalas (2004) Five Years of Continuous-Time Random Walks in Econophysics. Proceedings of WEHIA 2004, A. Namatame (ed.), Kyoto.

[58] R.L. Schilling, Growth and Hölder conditions for sample paths of Feller proceses. Probability Theory and Related Fields 112, 565-611 (1998) MR:1664705 (99m:60131)

[59] R. Schumer, D.A. Benson, M.M. Meerschaert and S. W. Wheatcraft (2001) Eulerian derivation of the fractional advection-dispersion equation. J. Contaminant Hydrol., 48, 69-88.

[60] Z. Shi and M. Yor, Integrability and lower limits of the local time of iterated Brownian motion, Studia Sci. Math. Hungar. 33 (1997), no. 1-3, 279-298. MR1454115 (98i:60069)

[61] M. Shlesinger, J. Klafter and Y.M. Wong (1982) Random walks with infinite spatial and temporal moments. J. Statist. Phys. 27, 499-512. MR659807 (83e:82066)

[62] Y.G. Sinai (1982) The limiting behavior of a one-dimensional random walk in a random medium. Theor. Probab. Appl. 27, 256-268. MR657919 (83k:60078)

[63] I.M. Sokolov and J. Klafter (2005) From Diffusion to Anomalous Diffusion: A Century after Einstein's Brownian Motion. Chaos 15, 6103-6109. MR2150232 (2006d:82060) 
[64] Y. Xiao, Local times and related properties of multi-dimensional iterated Brownian motion, J. Theoret. Probab. 11 (1998), no. 2, 383-408. MR.1622577 (99g:60136)

[65] G. Zaslavsky, Fractional kinetic equation for Hamiltonian chaos. Chaotic advection, tracer dynamics and turbulent dispersion. Phys. D 76 (1994), 110-122. MR.1295881 (95h:58120)

Department of Mathematics and Statistics, University of Otago, P.O. Box 56, Dunedin, New Zealand

E-mail address: bbaeumer@maths.otago.ac.nz

Department of Probability and Statistics, Michigan State University, East Lansing, MiCHIGAN 48823

E-mail address: mcubed@stt.msu.edu

Department of Probability and Statistics, Michigan State University, East Lansing, MichigAn 48823

E-mail address: nane@stt.msu.edu

Current address: Department of Mathematics and Statistics, Auburn University, 340 Parker Hall, Auburn, Alabama 36849

E-mail address: nane@auburn.edu 